\title{
Evaluation of serum s-IgE/total IgE ratio in predicting clinical response to allergen-specific immunotherapy
}

\author{
Gabriele Di Lorenzo, MD, ${ }^{a}$ Pasquale Mansueto, MD, ${ }^{a}$ Maria Luisa Pacor, MD, ${ }^{b}$ Manfredi Rizzo, MD, ${ }^{a}$ \\ Francesco Castello, MD, ${ }^{a}$ Nicola Martinelli, MD, ${ }^{b}$ Vito Ditta, MD, ${ }^{\text {a }}$ Claudia Lo Bianco, MD, ${ }^{a}$ \\ Maria Stefania Leto-Barone, MD, ${ }^{a}$ Alberto D'Alcamo, MD, ${ }^{a}$ Gaetana Di Fede, MD, ${ }^{c}$ Giovam Battista Rini, MD, \\ and Anne Marie Ditto, MD ${ }^{\mathbf{d}}$ Palermo and Verona, Italy, and Chicago, Ill
}

Background: To date, no predictive tests for the clinical response to allergen-specific immunotherapy (ASI) are available.

Therefore an in vivo or in vitro test would be of great value. Objective: We sought to evaluate pretreatment parameters used in diagnosing allergic rhinitis and determining serum specific IgE (s-IgE) levels, serum total IgE (t-IgE) levels, and blood eosinophil counts and to identify whether can be used to predict clinical improvement in monosensitized patients with allergic rhinitis with or without asthma treated with immunotherapy. Methods: We analyzed 279 patients who had undergone 4 years of ASI administered either by means of the subcutaneous

immunotherapy (76 patients) or sublingual immunotherapy (203 patients) routes. Serum t-IgE and s-IgE levels, blood eosinophil counts, and serum s-IgE/t-IgE ratios were calculated and tested for correlation with clinical response to ASI. Receiver operating characteristic curves were determined. Predicted probabilities and predictive areas under the curve were calculated.

Results: The clinical response to ASI was effective in 145 $(52.0 \%)$ of 279 total patients, $42(55.2 \%)$ of 76 patients treated with subcutaneous immunotherapy, and $103(50.7 \%)$ of 203 patients treated with sublingual immunotherapy. A significant correlation was found between the serum s-IgE/t-IgE ratio and the clinical response to ASI, with high ratios $(>16.2)$ associated with an effective response. The sensitivity and specificity of the area under the curve of the ratio were higher than those of serum s-IgE and t-IgE alone.

Conclusion: The calculation of the serum s-IgE/t-IgE ratio for predicting the clinical response to ASI offers an advantage over measuring t-IgE and s-IgE levels in monosensitized patients for the following allergens: grass, Parietaria judaica,

From ${ }^{a}$ Dipartimento di Medicina Clinica e delle Patologie Emergenti and ${ }^{\mathrm{c}}$ Dipartimento di Discipline Chirurgiche ed Oncologiche, Università degli Studi di Palermo; ${ }^{\mathrm{b}}$ Dipartimento di Medicina Clinica e Sperimentale, Università degli Studi di Verona; and ${ }^{\mathrm{d}}$ the Department of Medicine, Division of Allergy-Immunology, Northwestern University Feinberg School of Medicine, Chicago.

Supported by grants from the Ministero Italiano dell'Università e della Ricerca (MIUR; fondi ex 60\%) to Gabriele Di Lorenzo. Anne Marie Ditto is supported by the Ernest S. Bazley Grant

Disclosure of potential conflict of interest: A. M. Ditto is a consultant for Dey Pharmaceuticals and Abbott Pharmaceuticals and has provided legal consultation/ expert witness testimony in cases related to Stevens-Johnson syndrome and latex allergy. The rest of the authors have disclosed that they have no conflict of interest.

Received for publication February 19, 2008; revised February 3, 2009; accepted for publication February 9, 2009.

Available online April 8, 2009.

Reprint requests: Gabriele Di Lorenzo, MD, Dipartimento di Medicina Clinica e delle Patologie Emergenti, Via del Vespro, 141-90127 Palermo, Italy. E-mail: dilo601@ unipa.it.

$0091-6749 / \$ 36.00$

(c) 2009 American Academy of Allergy, Asthma \& Immunology

doi:10.1016/j.jaci.2009.02.012
Olea europea, and house dust mite. (J Allergy Clin Immunol 2009;123:1103-10.)

Key words: Allergen-specific immunotherapy, total IgE, specific IgE, blood eosinophil counts, serum-specific IgE/serum total IgE ratio, receiver operating characteristic curve

Allergen-specific immunotherapy (ASI) is the practice of administering gradually increasing doses of allergens (allergen extracts or vaccines) to reduce allergic symptoms and the need for medication resulting from exposure to a specific allergen. ${ }^{1,2}$ Subcutaneous immunotherapy (SCIT) was introduced into clinical practice early in the 20th century. ${ }^{3}$ In 1986 , sublingual immunotherapy (SLIT) was introduced. ${ }^{4,5}$ Double-blind placebo-controlled trials and meta-analyses confirm the efficacy, safety, and indications and contraindications of both SCIT and SLIT. $^{6-8}$ However, the efficacy of ASI is still debated, despite solid documentation. ASI, in practice, is indicated for patients for whom the causative role of the allergen is well documented. This is determined most commonly in vivo in skin tests (eg, skin prick testing) and in vitro in quantitative assays for serum specific IgE (s-IgE; eg, Unicap 100; Phadia, Uppsala, Sweden). It is unclear how serum total $\operatorname{IgE}(\mathrm{t}-\mathrm{IgE})$ measurements, alone or in relation to s-IgE measurements, or blood eosinophil (b-eos) counts should be interpreted, as well as what role they should play in selecting patients for ASI in clinical practice. Furthermore, the significance of serum s-IgE levels might vary depending on the level of $\mathrm{t}-\mathrm{IgE}$.

In this study we report the clinical results of 279 patients who received 4 years of ASI in a clinical setting administered either by means of SCIT or SLIT. We retrospectively examined the relationship of the following parameters determined at the time of diagnosis: diameter of wheal induced by the allergen, serum t-IgE levels, serum s-IgE levels, b-eos counts, and clinical response to ASI. We used receiver operating characteristic (ROC) curves to determine the sensitivity, specificity, and predicted values for wheal diameter, serum s-IgE level, serum t-IgE level, serum s-IgE/ $\mathrm{t}-\mathrm{IgE}$ ratio, and b-eos count, all obtained at the time of diagnosis, in predicting one's response to ASI.

\section{METHODS \\ Patients}

We retrospectively analyzed monosensitized adult patients consecutively referred to the Outpatient Allergy Units of the Dipartimento di Medicina Clinica e delle Patologie Emergenti of the University of Palermo, Italy, and of the Dipartimento di Medicina Clinica e Medicina Sperimentale of the University of Verona, Italy, between January 1995 and December 2000 for evaluation of their allergic rhinitis with or without asthma symptoms. All patients underwent ASI as part of the therapy for allergic rhinitis. Patients did 


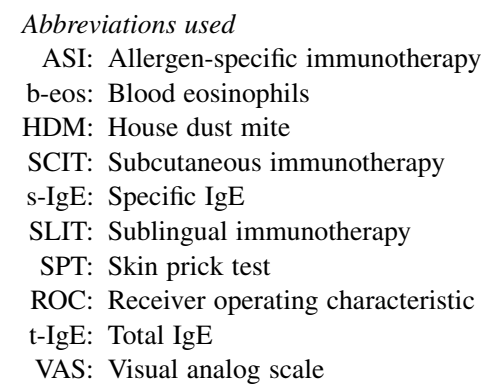

not have other allergic diseases, such as atopic dermatitis, eczema, or nasal polyps.

A total of 279 subjects (120 male and 159 female subjects), with ages ranging between 18 and 56 years (mean age, $29.5 \pm 8.1$ years) were selected: 126 subjects from Palermo and 153 subjects from Verona, respectively.

All patients presented with a clinical diagnosis of allergic rhinitis with or without asthma based on patient-reported symptoms, physical examination, and a normal baseline lung function test (baseline $\mathrm{FEV}_{1} \geq 80 \%$ of predictive value). All patients has positive skin test responses for 1 of 11 common airborne allergens, as determined by using the skin prick test method, with monosensitization to 1 of the following: grass, Parietaria judaica, Olea europea, or house dust mite (HDM). All were treated with ASI for at least 4 years administered either as SCIT or SLIT. At the time of diagnosis, blood was collected for analysis of serum t-IgE levels, s-IgE levels, and b-eos counts. Family history of atopy, as well as history of smoking and onset of respiratory symptoms, was obtained from each patient. Clinical evaluations were done at baseline and once a year for the 4-year period of ASI. Evaluations included rhinoscopy and spirometry (if asthmatic), symptom scoring with the visual analog scale (VAS), and medication use, referring to the previous year. Evaluations were conducted at the end of the season for the 3 outdoor allergens and at the end of February for Dermatophagoides pteronyssinus and Dermatophagoides farinae. More precisely, we performed a clinical evaluation and administered the VAS for symptoms in June for patients allergic to grass and $O$ europe $a$ and in September for patients allergic to $P$ judaica. For patients allergic to $D$ pteronyssinus and $D$ farinae, we performed a clinical evaluation and administered the VAS (in February) because the symptoms were worse in autumn, after vacations, and during winter (for more information, see the Methods section in this article's Online Repository at www.jacionline.org).

Because ASI is commercially available and was prescribed for indications that are recognized both nationally and internationally, our ethics committees required written informed consent for the diagnostic tests only, and this was obtained from each patient. ${ }^{8-10}$

\section{Immunotherapy intervention}

The route of administration of ASI (SCIT or SLIT) was chosen by the patient to ensure compliance. Each patient received the maximum tolerated dose, per the manufacturers' recommendations, for both SCIT and SLIT. All the patients in this study tolerated the maximum dose indicated by the manufacturers' recommendations as follows. For SCIT, the maintenance dose was $0.8 \mathrm{~mL}$, which corresponds to $1.6 \mu \mathrm{g}$ of the major allergen of grass, $0.4 \mu \mathrm{g}$ of the major allergen of $P$ judaica, $9.6 \mu \mathrm{g}$ of the major allergen of $O$ europea, and $3.84 \mu \mathrm{g}$ of the major allergens of $D$ pteronyssinus and $D$ farinae. On the other hand, for SLIT, the maintenance dose was 60 drops $(1$ drop $=25 \mu \mathrm{L})$ administered 3 times a week on alternate days, which corresponds to 28.2 $\mu \mathrm{g}$ of the major allergen of grass, $6.6 \mu \mathrm{g}$ of the major allergen of $P$ judaica, $169.2 \mu \mathrm{g}$ of the major allergen of $O$ europea, and $67 \mu \mathrm{g}$ of the major allergens of $D$ pteronyssinus and $D$ farinae. These doses were administered monthly. Immunotherapy with grass, $P$ judaica, and $O$ europea pollen was not interrupted during the spring, but the dose was halved (see the Methods section in this article's Online Repository).
TABLE I. Characteristics of cohort study patients examined in the 2 centers

\begin{tabular}{|c|c|}
\hline No. of patients & 279 \\
\hline Age $(y)^{*}$ & $29.5(28.6-30.5)$ \\
\hline Male/female sex & $120 / 159$ \\
\hline SCIT/SLIT & $76 / 203$ \\
\hline Atopic family history (yes/no) & $116 / 163$ \\
\hline Passive smoking (yes/no) & $123 / 156$ \\
\hline Rhinitis/rhinitis plus asthma & $152 / 127$ \\
\hline Onset of symptoms $(\mathrm{y})^{*}$ & $7.1(6.4-7.7)$ \\
\hline Pollens/HDM sensitization (no) & $106 / 173$ \\
\hline Response to ASI (effective/ineffective) & $145 / 134$ \\
\hline SPT to allergen $(\mathrm{mm}) \dagger$ & $5.4(5.3-5.5)$ \\
\hline Serum t-IgE level $(\mathrm{kU} / \mathrm{L}) \dagger$ & $139.3(121.4-159.9)$ \\
\hline Serum s-IgE level $(\mathrm{kAU} / \mathrm{L}) \dagger$ & $20.2(17.6-23.0)$ \\
\hline b-eos Counts (cells $\left.\times 10^{-3} \mu \mathrm{L}\right) \dagger$ & $0.35(0.34-0.37)$ \\
\hline Serum s-IgE/t-IgE* ratio & $14.4(12.3-16.9)$ \\
\hline
\end{tabular}

*Mean $(95 \% \mathrm{CI})$.

$\dagger$ Geometric mean after logarithmic transformation $(95 \% \mathrm{CI})$.

\section{Assessment of symptoms and medication use}

The effectiveness of ASI was evaluated on the basis of clinical response (reduction in nasal and pulmonary symptoms) and reduction of the pharmacotherapy taken on an as-needed basis (eg, oral second-generation $\mathrm{H}_{1}$-antihistamine for rhinitis and inhaled short-acting $\beta_{2}$-agonist for asthma symptoms). ${ }^{10,11}$ Patients were not previously treated with nasal or inhaled corticosteroids.

VAS. To evaluate symptom response to ASI and reduction of pharmacotherapy, we used a VAS sufficiently sensitive to detect changes in symptom severity (see the Methods section in this article's Online Repository). ${ }^{12,13}$

The response to ASI was determined clinically by asking the patient the following: "Do you feel better than you did before therapy?" The answer could be one of the following:

1. I have not noticed any improvement since I have used ASI.

2. I noticed a worsening of symptoms since I have used ASI.

3. I noticed that my symptoms improved since I have used ASI.

When we analyzed the 1624 ASI VAS responses throughout the 4-year study, we noticed that patients who stated improvement of symptoms during immunotherapy indicated a reduction in their rhinitis (or asthma) VAS values of at least $30 \%$ compared with their baseline VAS values before starting immunotherapy at the time of diagnosis. To be sure of the results, for each subject, we used all the ASI values obtained for each year, calculated the mean, and then subtracted the baseline VAS (VASb) from the mean of the VAS obtained over 4 years. Then we divided the result by the baseline VAS value and multiplied by 100 , as shown by the following formula:

$$
\text { VAS decrease }(\%)=\frac{(\text { Mean } V A S-V A S b)}{V A S b} \times 100
$$

We evaluated the ASI as effective if the mean of the VAS scores for the 4 years showed a $30 \%$ decrease with respect to the value of the VAS score before ASI by using the formula reported above and if the values of the rescue medications indicated at the third and fourth years of ASI were 0 or 1 . In all other cases, ASI was evaluated as ineffective. Symptom scoring and the medication used were reviewed by a physician (PM for Palermo and NM for Verona).

Spirometry. $\mathrm{FEV}_{1}$ and forced expiratory vital capacity were measured with a Gould 2400 automated system (Sensormedics BV, Bilthoven, Netherlands) by using the highest of 3 successive measurements, provided that the difference between measurements was within $100 \mathrm{~mL}$. Spirometric results were followed each year for 4 years. There was no significant change in $\mathrm{FEV}_{1}$ or forced expiratory vital capacity (all $>80 \%$ of predicted value) in any patient, irrespective of whether the patient responded clinically to ASI. 
TABLE II. Characteristics of the cohort study patients in respect to effective or ineffective clinical response to ASI

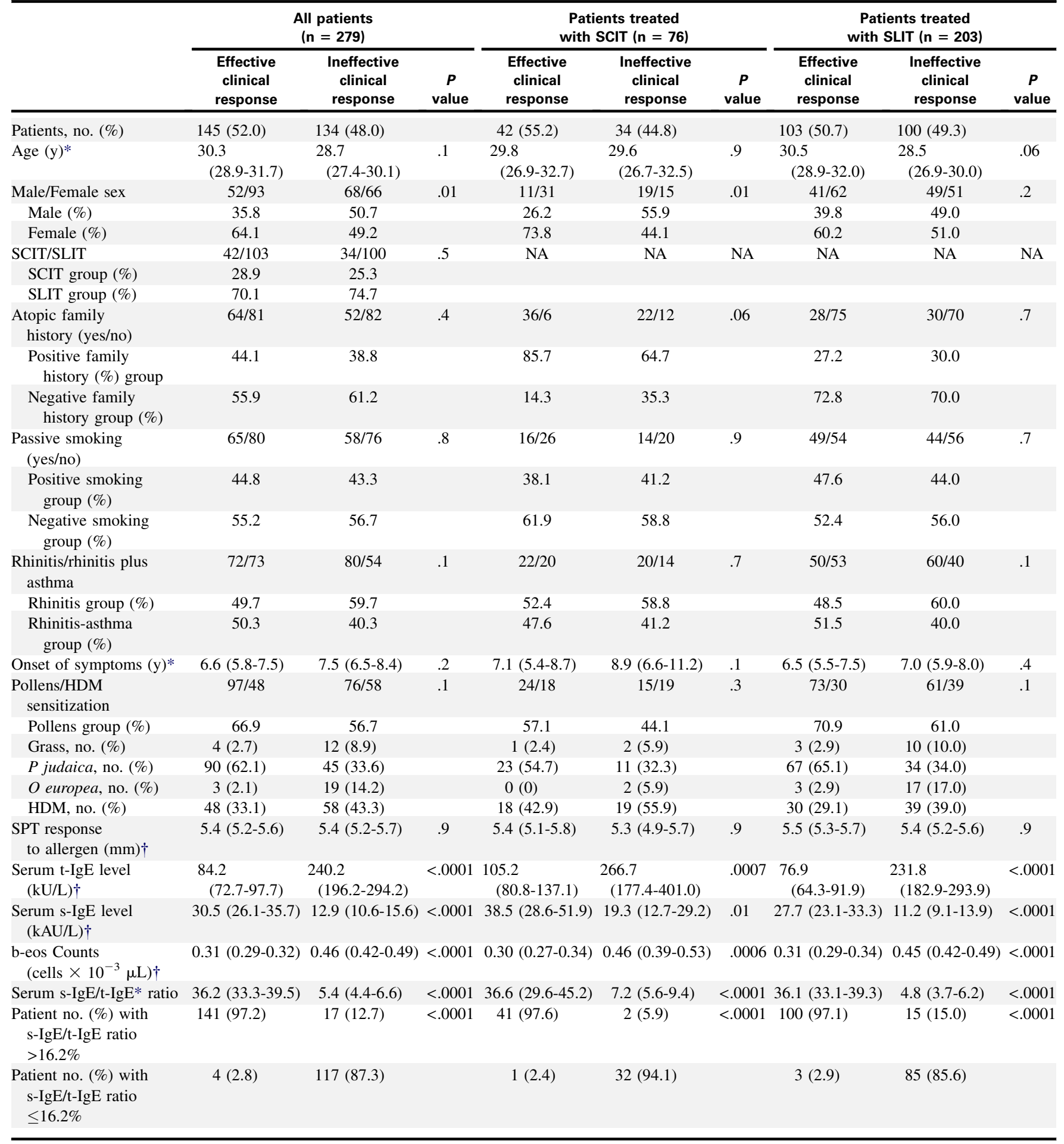

NA, Not applicable.

* Mean $(95 \% \mathrm{CI})$.

†Geometric mean after logarithmic transformation (95\% CI).

Skin prick tests. Skin prick tests with a standard aeroallergen panel (Alk-Abelló, Milan, Italy) were performed and evaluated on the volar aspect of the forearm after withholding antihistamines for at least 5 days. The panel included the following extracts: pollens (grass [Phleum pratense], mugwort [Artemisia vulgaris], pellitory of the wall or sticky weed $[P$ judaica], and trees [O europea and Cupressus species]), HDM (D pteronyssinus and D farinae), molds (Alternaria alternata and Aspergillus fumigatus), and animal dander (cat and dog), as well as a negative control (glycerinated saline) and a positive control (histamine, $10 \mathrm{mg} / \mathrm{mL}$ ). A positive response was defined as any wheal with a diameter $3 \mathrm{~mm}$ larger than that elicited by the negative control 15 minutes after application. ${ }^{14}$ The wheal diameters were reported for each patient. 
TABLE III. Characteristics of patients with effective and ineffective responses to ASI in respect to symptoms

\begin{tabular}{|c|c|c|c|c|c|c|}
\hline & \multicolumn{3}{|c|}{$\begin{array}{l}\text { Patients with } \\
\text { rhinitis }(\mathrm{n}=152)\end{array}$} & \multicolumn{3}{|c|}{$\begin{array}{l}\text { Patients with } \\
\text { rhinitis plus asthma }(n=127)\end{array}$} \\
\hline & $\begin{array}{l}\text { Effective clinical } \\
\text { response }\end{array}$ & $\begin{array}{l}\text { Ineffective clinical } \\
\text { response }\end{array}$ & $P$ value & $\begin{array}{l}\text { Effective clinical } \\
\text { response }\end{array}$ & $\begin{array}{l}\text { Ineffective clinical } \\
\text { response }\end{array}$ & $P$ value \\
\hline Age $(y)^{*}$ & $29.3(27.5-31.2)$ & $28.3(26.6-30.0)$ & .4 & $31.3(29.2-33.3)$ & $29.4(27.2-31.6)$ & .2 \\
\hline Male/female sex & $25 / 47$ & $41 / 39$ & .04 & $27 / 46$ & $27 / 27$ & .1 \\
\hline Male (\%) & 34.7 & 51.2 & & 37.0 & 50.0 & \\
\hline SCIT group (\%) & 30.6 & 25.0 & & 27.4 & 26.0 & \\
\hline SLIT group (\%) & 69.4 & 75.0 & & 72.6 & 74.0 & \\
\hline Atopic family history (yes/no) & $36 / 36$ & $34 / 46$ & .4 & $28 / 45$ & $18 / 36$ & .5 \\
\hline Positive family history group (\%) & 50.0 & 42.5 & & 38.4 & 33.3 & \\
\hline Negative family history group (\%) & 40.0 & 57.5 & & 61.6 & 66.7 & \\
\hline Passive smoking (yes/no) & $30 / 42$ & $33 / 47$ & .9 & $35 / 38$ & $25 / 29$ & .8 \\
\hline Pollens group $(\%)$ & 62.5 & 57.5 & & 71.2 & 55.6 & \\
\hline HDM group (\%) & 37.5 & 42.5 & & 28.8 & 44.4 & \\
\hline Grass, no. (\%) & $1(1.4)$ & $8(10)$ & & $3(4.1)$ & $4(7.4)$ & \\
\hline$P$ judaica, no. (\%) & $43(59.7)$ & $24(30)$ & & $47(64.4)$ & $21(38.9)$ & \\
\hline O europea, no. (\%) & $1(1.4)$ & $14(17)$ & & $2(2.7)$ & $5(9.3)$ & \\
\hline HDM, no. $(\%)$ & $27(37.5)$ & $34(43)$ & & $21(28.8)$ & $24(44.4)$ & \\
\hline SPT response to allergen $(\mathrm{mm}) \dagger$ & $5.3(5.0-5.6)$ & $5.3(5.1-5.6)$ & .7 & $5.4(5.1-5.6)$ & $5.3(5.0-5.6)$ & .8 \\
\hline Serum $\mathrm{t}-\mathrm{IgE}$ level $(\mathrm{kU} / \mathrm{L}) \dagger$ & $84.2(68.7-103.2)$ & $206.4(161.5-263.8)$ & $<.0001$ & $84.2(67.6-104.9)$ & $300.8(212.3-428.2)$ & $<.0001$ \\
\hline Serum s-IgE level $(\mathrm{kAU} / \mathrm{L}) \dagger$ & $30.1(24.6-37.5)$ & $10.0(7.9-12.7)$ & $<.0001$ & $30.9(24.6-38.7)$ & $18.6(13.6-25.3)$ & .01 \\
\hline b-eos Counts $\left(\right.$ cells $\left.\times 10^{-3} \mu \mathrm{L}\right) \dagger$ & $0.31(0.29-0.33)$ & $0.40(0.37-0.42)$ & $<.0001$ & $0.30(0.28-0.33)$ & $0.43(0.40-0.47)$ & $<.0001$ \\
\hline Serum s-IgE/t-IgE* ratio & $41.5(36.9-48.1)$ & $9.9(7.6-12.2)$ & $<.0001$ & $39.8(35.1-44.5)$ & $8.1(8.5-9.7)$ & $<.0001$ \\
\hline Patient no. (\%) with s-IgE/t-IgE ratio $>16.2 \%$ & $70(97.2)$ & $10(12.5)$ & $<.0001$ & $71(97.3)$ & $7(13.0)$ & $<.0001$ \\
\hline
\end{tabular}

*Mean (95\% CI).

$\dagger$ Geometric mean after logarithmic transformation $(95 \% \mathrm{CI})$.

\section{Serum t-IgE and s-IgE levels}

A blood sample was processed at the time of diagnosis and before ASI. Serum t-IgE and s-IgE levels were determined by using the Unicap 100 with the fluoroimmunoassay technique (Phadia, Uppsala, Sweden), according to the manufacturer's instructions. Results for both serum t$\operatorname{IgE}$ and s-IgE measurements were expressed in kilounits per liter and equilibrated against the World Health Organization standard for IgE: $1 \mathrm{kU}$ for $\mathrm{t}-\mathrm{IgE}$ and $1 \mathrm{kAU}$ for $\mathrm{s}-\mathrm{IgE}$ was equal to $2.4 \mathrm{ng} / \mathrm{mL}$, respectively. Serum t-IgE levels were determined with a detection limit of $2 \mathrm{kU} / \mathrm{L}$ and an upper limit of $5000 \mathrm{kU} / \mathrm{L}$. Serum s-IgE levels were determined with a detection limit of $0.35 \mathrm{kAU} / \mathrm{L}$ and an upper limit of $100 \mathrm{kAU} / \mathrm{L}$. In all patients the s-IgE level was measured for the same allergens used in the skin prick tests (11 allergens). ${ }^{15,16}$ All patients were monosensitized (had a positive skin prick test response for only 1 allergen and presented with $\mathrm{s}$-IgE for the same allergen). For the calculation of the ratio, we used the following formula:

$$
s-\operatorname{Ig} E / t-\operatorname{IgE} \text { ratio }=\frac{s-\operatorname{Ig} E}{t-\operatorname{Ig} E} \times 100
$$

Peripheral b-eos counts. Absolute peripheral b-eos counts were determined before ASI with a Technicon-H1 blood cell counter (Bayer, Leverkusen, Germany), with the normal range being 0.10 to 0.40 cells $/ 10^{-3} \mu \mathrm{L} .{ }^{17}$

\section{Statistical analysis}

Statistical analysis was performed with the SYSTAT 10 software package (Systat Software, Inc, San Jose, Calif). Data were reported as the arithmetic
TABLE IV. Multivariate regression using the response to ASI as variable-dependent

\begin{tabular}{lrl}
\hline Independent variables & $\boldsymbol{r}$ & $\boldsymbol{P}$ value \\
\hline Age (y) & 0.095 & NS \\
Male/female sex & -0.150 & NS \\
SCIT/SLIT & 0.040 & NS \\
Atopic family history & 0.054 & NS \\
Rhinitis/rhinitis plus asthma & 0.101 & NS \\
Onset of symptoms (y) & -0.075 & .007 \\
Pollens/HDM sensitization & 0.105 & NS \\
Serum s-IgE/t-IgE ratio & 0.723 & $<.0001$ \\
Serum t-IgE level & -0.342 & NS \\
Serum s-IgE level & 0.316 & .007 \\
b-eos Counts & -0.429 & $<.0001$ \\
SPT response to allergens (mm) & 0.009 & NS \\
\hline
\end{tabular}

$N S$, not significant.

mean and $95 \%$ CI for the mean when the distribution of the data was normal. When normality was rejected, data were examined after logarithmic transformation and presented as the geometric mean and the 95\% CI for the geometric mean. We used the Kolmogorov-Smirnov test to evaluate the normal distribution. Group comparison tests were performed with the 2 -sided $t$ test. Categorical variables between groups were compared by using the $\chi^{2}$ test. An effective or ineffective response to ASI was assessed by examining the area under the ROC curves (c-statistic). Models with an ROC area of at least 0.80 


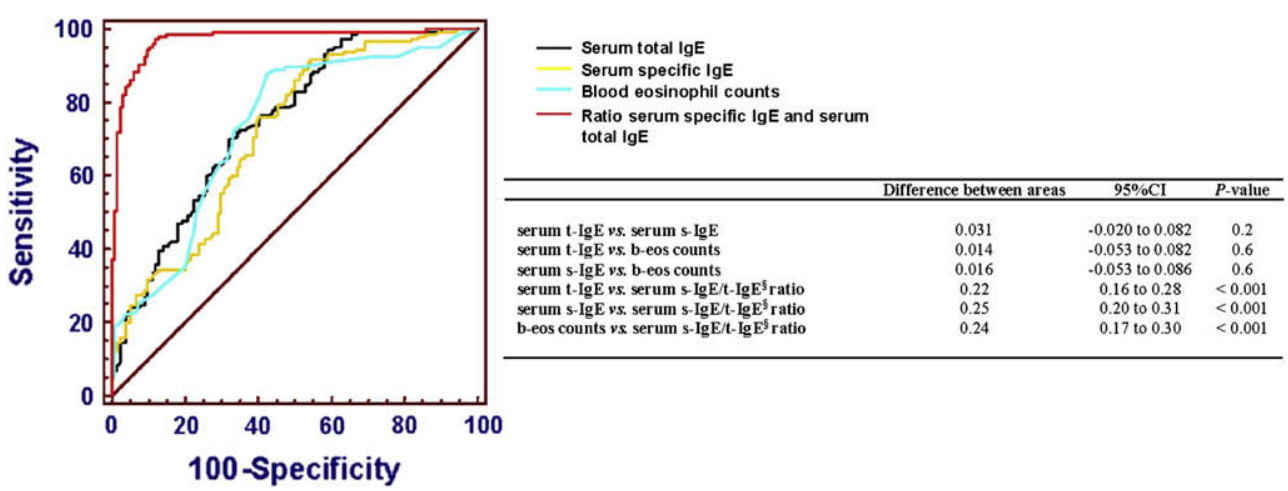

FIG 1. ROC curves obtained with serum t-lgE levels (decision point, $\leq 133 \mathrm{kU} / \mathrm{L}$; sensitivity, $69.7 \%$; and specificity, 67.9\%), serum s-IgE levels (decision point, $9.5 \mathrm{kAU} / \mathrm{L}$; sensitivity, $91.0 \%$; specificity, $46.3 \%$ ), b-eos counts (decision point, 0.43 cells $\times 10^{-3} \mu \mathrm{L}$; sensitivity, $87.6 \%$; specificity, $57.5 \%$ ), and serum s-lgE/serum t-lgE ratios (decision point, $16.2 \%$; sensitivity, $97.2 \%$; specificity, $88.1 \%$ ) by plotting sensitivity in patients with an effective response to ASI versus 100-specificity in patients with an ineffective response to ASI.

were considered to have good predictive value. ${ }^{18}$ For all analyses, a $P$ value of less than .05 was considered statistically significant.

\section{RESULTS}

We examined a total of 120 male and 159 female subjects (mean age, $29.5 \pm 8.1$ years) meeting the criteria outlined above. One hundred fifty-two patients were affected by rhinitis alone, and 127 were affected by rhinitis plus asthma. Sixteen patients were monosensitized to grass, 135 to $P$ judaica, 22 to $O$ europea, and 106 to HDM. Two hundred three $(72.8 \%)$ patients were treated with SLIT, and $76(27.2 \%)$ were treated with SCIT. Response to ASI was considered effective in $145(52.0 \%)$ patients: 4 sensitive to grass, 90 sensitive to $P$ judaica, 3 sensitive to $O$ europea, and 48 sensitive to HDM. ASI was considered clinically ineffective in 134 (48.0\%) patients: 12 sensitive to grass, 45 sensitive to $P$ judaica, 19 sensitive to $O$ europea, and 58 sensitive to HDM.

Patient characteristics are reported in Table I. Table II compares patients with an effective clinical response to ASI with those with an ineffective response, both as a group and according to the type of ASI (SLIT and SCIT). When comparing all the study patients, those who had an effective response to ASI differed significantly from those with an ineffective response in the following characteristics: sex (female subjects better than male subjects, $P=.01$ ), serum t-IgE levels (low levels better than high levels, $P<.0001$ ), serum s-IgE levels (high levels better than low levels, $P<.0001$ ), b-eos counts (low counts better than high counts, $P<.0001$ ), and serum s-IgE/t-IgE ratios (high ratios better than low ratios, $P<.0001)$. Between patients with effective and ineffective clinical responses to SCIT, significant differences were found with regard to the following characteristics: sex (female subjects better than male subjects, $P=.01$ ), serum t-IgE levels (low levels better than high levels, $P=.007$ ), serum s-IgE levels (high levels better than low levels, $P=.01$ ), b-eos counts (low counts better than high counts, $P<.0001$ ), and serum s-IgE/t-IgE ratios (high ratios better than low ratios, $P<.0001$ ). Significant differences were found between patients with effective and ineffective clinical responses to SLIT with regard to serum t-IgE levels (low levels better than high levels, $P<$ .0001 ), serum s-IgE levels (high levels better than low levels, $P<.0001$ ), b-eos counts (low counts better than high counts, $P<.0001$ ), and serum s-IgE/t-IgE ratios (high ratios better than low ratios, $P<.0001)$. We did not find a relationship among allergen dosing, total dose received, and clinical response because all the patients within each group received an equal dose.

Table III shows the characteristics of patients with rhinitis only and those with rhinitis and asthma symptoms. For patients with rhinitis only, significant differences were found between those with effective and ineffective clinical responses to ASI with regard to serum t-IgE levels (low levels better than high levels, $P \leq$ .0001 ), serum s-IgE levels (high levels better than low levels, $P \leq .0001$ ), b-eos counts (low counts better than high counts, $P<.0001$ ), and serum s-IgE/t-IgE ratios (high ratios better than low ratios, $P<.0001)$. For patients with rhinitis and asthma symptoms, significant differences were found between patients with effective and ineffective clinical responses to ASI with regard to serum t-IgE levels (low levels better than high levels, $P \leq$ .0001 ), serum s-IgE levels (high levels better than low levels, $P \leq .01$ ), b-eos counts (low counts better than high counts, $P<.0001$ ), and serum s-IgE/t-IgE ratios (high ratios better than low ratios, $P<.0001$ )

Serum t-IgE and s-IgE levels correlated with age (both showing), whereas the s-IgE/t-IgE ratio was not affected by age. Regression analysis showed that t-IgE levels $(r=-0.13$ [95\% CI, -0.24 to -0.01$], P=.02)$ and s-IgE levels $(r=$ -0.17 [ $95 \% \mathrm{CI},-0.28$ to -0.05$], P=.004)$ were inversely related to age in years, whereas s-IgE/t-IgE ratios did not correlate with age $(P=.3)$. In addition, using multivariate regression analysis, we found that response to ASI is independently related only to onset of symptoms, s-IgE/t-IgE ratios, s-IgE levels, and b-eos counts, again confirming that response to ASI is not related to age (Table IV).

Fig 1 shows the sensitivity and specificity obtained by calculating ROC curves for serum t-IgE levels, serum s-IgE levels, b-eos counts, and serum s-IgE/t-IgE ratios and the pairwise comparisons of the ROC curves. The area under the curve was 0.74 for serum t-IgE levels (95\% CI, 0.69-0.79), 0.71 for serum s-IgE levels (95\% CI, 0.65-0.76), 0.73 for b-eos counts (95\% CI, 0.67-0.78), and 0.97 for serum s-IgE/t-IgE ratios (95\% CI, 0.94-0.98). Significant differences were found between the s-IgE/t-IgE ratio and $\mathrm{t}$-IgE levels alone $(P<.001)$, s-IgE levels alone $(P<.001)$, and b-eos counts $(P<.001)$. No other significant differences were found. 

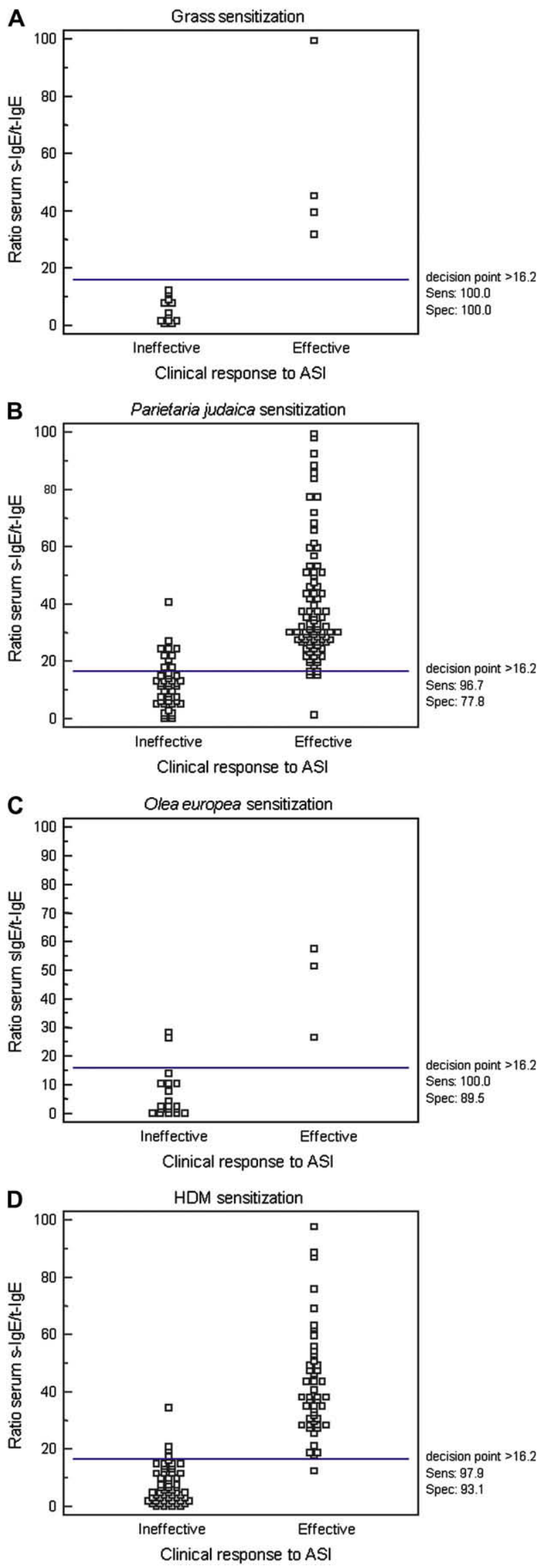

Our ROC analysis of the serum s-IgE/t-IgE ratio showed that a ratio of greater than $16.2 \%$ had the best sensitivity $(97.2 \%)$ and specificity ( $88.1 \%$ ) to predict effective ASI, regardless of whether SCIT or SLIT was used. The sensitivity and specificity of the decision point for a serum s-IgE/t-IgE ratio of greater than $16.2 \%$, as obtained from the ROC, was examined for each allergen used for immunotherapy. Results are as follows: sensitivity and specificity of $100 \%$ for grass; $96.7 \%$ and $77.8 \%$, respectively, for $P$ judaica; $100 \%$ and $89.5 \%$, respectively, for O europea; and $97.9 \%$ and $93.1 \%$, respectively, for HDM. This is shown in Fig 2. Fig 3 shows the means $( \pm$ SD) of VAS scores with regard to rhinitis and asthma symptoms, respectively. Of note, rescue medication use decreased with improvement in symptoms, as reflected in the VAS. Likewise, medication use increased with worsened symptoms per the VAS (Tables E1-E3).

\section{DISCUSSION}

Our investigation shows that the serum s-IgE/t-IgE ratio significantly correlates with the clinical response to ASI administered through both the SCIT and SLIT routes. We considered the result of ASI to 4 aeroallergens epidemiologically very important in our geographic area (ie, grass, $P$ judaica, $O$ europea, and HDM) in a total of 279 patients. ${ }^{14}$ Tests to predict the outcome of ASI would be of tremendous help in daily practice, but data about this aspect of immunotherapy have not been reported. Therefore we evaluated clinical, functional, and laboratory characteristics evaluated at the time of clinical diagnosis and analyzed the variables that were statistically different between the patients with effective and ineffective clinical responses to ASI after 4 years. These were serum t-IgE levels, serum s-IgE levels, b-eos counts, and serum s-IgE/t-IgE ratios. We compared these variables, considering the clinical response to ASI, apart from the route of administration. We found that for all the allergens considered, a serum s-IgE/t-IgE ratio of greater than $16.2 \%$ correlated with an effective clinical response to ASI with a sensitivity of $97.2 \%$ and a specificity of $88.1 \%$. Our analyses demonstrated that the serum s$\mathrm{IgE} / \mathrm{t}-\mathrm{IgE}$ ratio is superior to both serum $\mathrm{t}-\mathrm{IgE}$ and $\mathrm{s}-\mathrm{IgE}$ levels alone in predicting clinical response to ASI. This is based on only 1 measurement of s-IgE and t-IgE levels, which is a limitation of the study. Both samples were drawn at the time of diagnosis when patients were manifesting symptoms and therefore during a time when the patient was presumed to have a high clinical exposure. Measurements were obtained uniformly for all subjects at the time of diagnosis, generally during peak allergen exposure, thus reflecting a real-life situation comparable with the usual clinical practice for the management of such patients. Certainly, these considerations might be too speculative, and further studies with serial evaluations of IgE concentrations, including measurements during the season to look for the ablation of the seasonal increase in allergen s-IgE levels, are needed to confirm our results. Measuring allergen-specific IgG levels would also be useful because these are classically used to demonstrate response to ASI. It has been reported that the percentage of allergen $\mathrm{s}-\mathrm{IgE}$ is approximately $25 \%$ of $\mathrm{t}-\mathrm{IgE}$. $^{19}$ Allergic inflammation is initiated by allergen molecules cross-linking their corresponding

FIG 2. Sensitivity (Sens) and specificity (Spec) calculated for each allergen used for ASI by using the ratio of serum s-lgE/serum $t-\lg E$ of $16.2 \%$ as the decision point. A, grass; B, Parietaria judaica; C, Olea europa; D, house dust mites (HDM). 

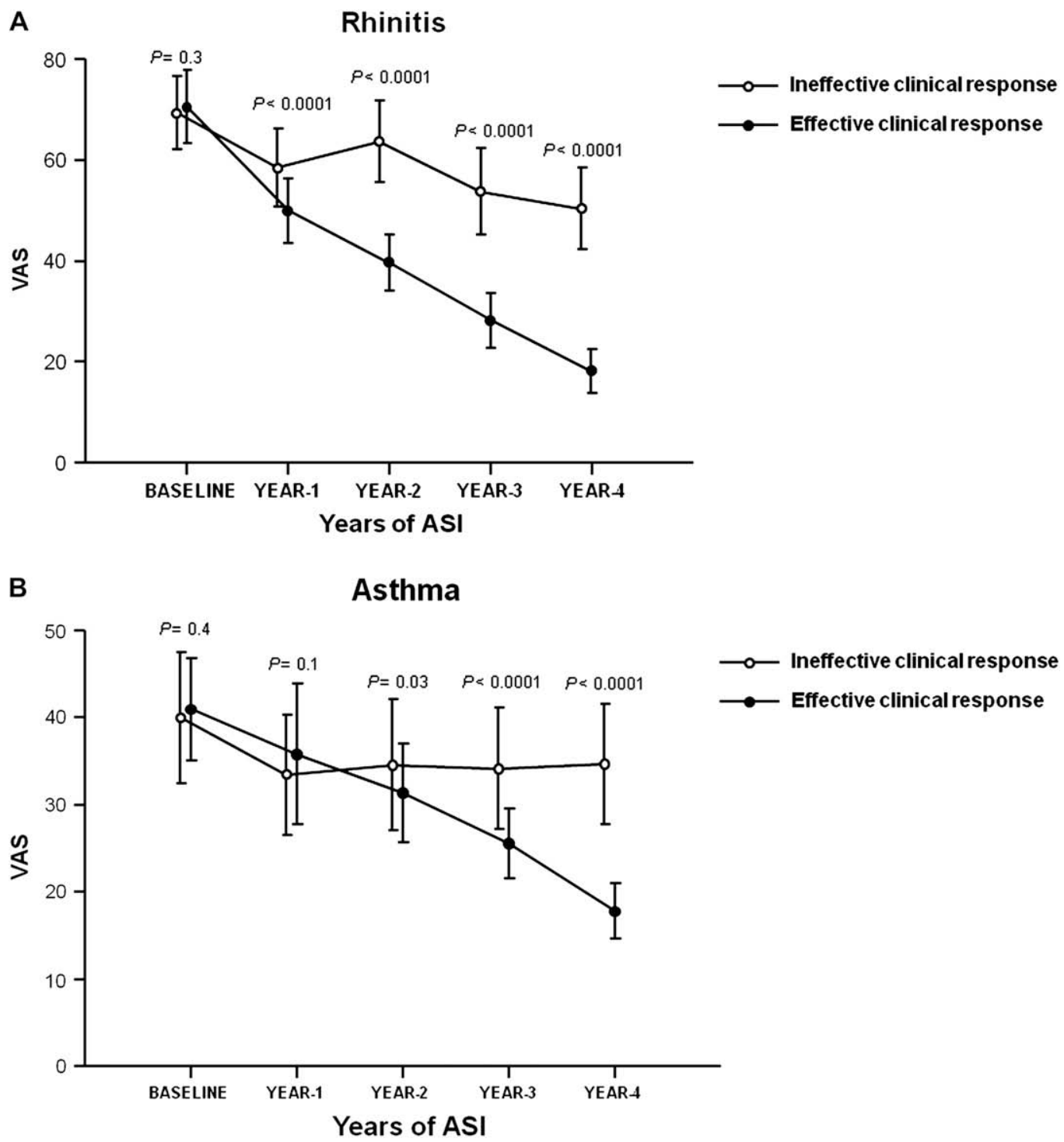

FIG 3. Graphic representation of means of VAS ( \pm SD) with regard to $\mathbf{A}$, rhinitis and $\mathbf{B}$, asthma symptoms, respectively.

receptor-bound $\mathrm{s}-\mathrm{IgE}$ on the mast cell and basophil surface. ${ }^{20,21}$ Although it is possible to quantify both serum t-IgE and s-IgE levels, serum t-IgE levels are not measured because they are not useful in the clinical diagnosis of allergy to common aeroallergens. ${ }^{15}$ Thus a higher s-IgE/t-IgE ratio might reflect a higher level of allergen s-IgE on the mast cell and basophil surface, whereas this probability is lower when the serum s-IgE/t-IgE ratio is low.

For this reason, it might be helpful to measure the serum t-IgE level and calculate the serum s-IgE/t-IgE ratio. In fact, as shown by the area under the curve values of the ROC, this ratio is at least as accurate, if not better, than serum t-IgE and s-IgE levels alone at predicting clinical response to ASI. Calculating a decision point for the ratio that represents a $95 \%$ predicted probability for an effective clinical response for all 4 allergens might improve the sensitivity and specificity compared with both serum s-IgE and tIgE levels alone. The predicted probability, calculated for each allergen studied, demonstrated that the cutoff level of greater than $16.2 \%$ showed both high specificity (between $77.8 \%$ for $P$ judaica and $100 \%$ for grass) and high sensitivity (between $96.7 \%$ for $P$ judaica and $100 \%$ for grass and $O$ europea).

The subjects in this study are monosensitized to the aeroallergens deemed most important in Italy. ${ }^{14}$ We cannot completely exclude the possibility of sensitization to allergens not routinely tested, nor can we apply the findings to monosensitized patients. However, it is likely that the amount of s-IgE in the serum reflects the degree of environmental exposure to individual allergens. Thus it is conceivable that the higher the s-IgE/t-IgE ratio, the more clinically relevant the allergen is for a particular patient. Likewise, the more clinically relevant the allergens included in the ASI, the more successful ASI will be. A possible mechanism is that ASI generates allergen-specific regulatory T cells, and generating regulatory $\mathrm{T}$ cells specific to the patient's dominant and most relevant allergen might help generate sufficient regulatory $\mathrm{T}$ cells in the respiratory mucosa to suppress bystander IgE responses to other less relevant allergens, resulting in an overall decrease in mucosal allergic inflammation. ${ }^{19}$ 
ASI has proved efficacy for a variety of inhalant allergens. However, the treatment is of long duration. Because not all patients benefit from treatment, it would be useful to be able to have specific criteria to determine those patients who might best benefit from this therapy. We found that the serum s-IgE/t- $\operatorname{IgE}$ ratio is the best predictor of clinical response to allergen-specific immunotherapy. Although the s-IgE/t-IgE ratio has been already described in the medical literature, to the best of our knowledge, this is the first study that demonstrates a possible role of s-IgE/t$\mathrm{IgE}$ ratio as a predictor of clinical response to allergen-specific immunotherapy. Our results are applicable only to patients monosensitized to these 4 allergens studied. In addition, the lack of precise data about pollen counts might be another limitation. However, there is no reason to think there was an important difference between years because the local registries for pollen counts did not reflect this. In addition, patients with no response to ASI required more rescue medication compared with those patients who responded, providing indirect evidence of adequate pollen counts.

Finally, the lack of a placebo is a limitation of this study. However, the aim of this study was not to perform a clinical trial that might be addressed in the future but to search for a potential explanation for why patients who have received ASI could have very different response rates and thus to search for possible predictors of such varied responses. Our study has a retrospective design and, consequently, has the common limitations of such types of studies. Nonetheless, we believe that these limitations do not significantly confound the main finding of our study. However, this needs to be further investigated, preferably through studies with a double-blind design, which is the gold standard for determining the efficacy of any therapy.

Clinical implications: The s-IgE/t-IgE ratio significantly correlated with the clinical response to ASI in monosensitized patients. This suggests that the s-IgE/t-IgE ratio can be used as a predictor of clinical response to immunotherapy.

\section{REFERENCES}

1. Bousquet J, Lockey R, Malling HJ. Allergen immunotherapy: therapeutic vaccines for allergic diseases. A WHO position paper. J Allergy Clin Immunol 1998;102:558-62.

2. Bousquet J, Van Cauwenberge P, Khaltaev N. Aria Workshop Group: World Health Organization. Allergic rhinitis and its impact on asthma. J Allergy Clin Immunol 2001;108(suppl):S147-334.

3. Noon L. Prophylactic inoculation against hay fever. Lancet 1911;1:1572-3.
4. Warner JO. Low dose sublingual therapy in patients with allergic rhinitis due to house dust mite. Clin Allergy 1986;16:387-8.

5. Scadding GK, Brostoff J. Low dose sublingual therapy in patients with allergic rhinitis due to house dust mite. Clin Allergy 1986;16:483-91.

6. Abramson MJ, Puy RM, Weiner JM. Allergen immunotherapy for asthma. Cochrane Database Syst Rev 2000;(2):CD001186.

7. Abramson M, Puy R, Weiner J. Immunotherapy in asthma: an updated systematic review. Allergy 1999;54:1022-41.

8. Wilson DR, Lima MT, Durham SR. Sublingual immunotherapy for allergic rhinitis: systematic review and meta-analysis. Allergy 2005;60:4-12.

9. Adkinson NF. Con: immunotherapy is not clinically indicated in the management of allergic asthma. Am J Respir Crit Care Med 2001;164:2140-2.

10. Bousquet J, Van Cauwenberge P. A critical appraisal of "evidence-based medicine” in allergy and asthma. Allergy 2004;59(suppl 78):12-20.

11. Canonica GW, Baena-Cagnani CE, Bousquet J, Bousquet PJ, Lockey RF, Malling $\mathrm{HJ}$, et al. Recommendations for standardization of clinical trials with Allergen Specific Immunotherapy for respiratory allergy. A statement of a World Allergy Organization (WAO) taskforce. Allergy 2007;62:317-24.

12. Ortolani C, Foresi A, Di Lorenzo G, Bagnato G, Bonifazi F, Crimi N, et al. A double-blind, placebo-controlled comparison of treatment with fluticasone propionate and levocabastine in patients with seasonal allergic rhinitis. FLNCO2 Italian Study Group. Allergy 1999;54:1173-80.

13. Di Lorenzo G, Mansueto P, Melluso M, Morici G, Norrito F, Esposito Pellitteri M, et al. Non-specific airway hyperresponsiveness in mono-sensitive Sicilian patients with allergic rhinitis. Its relationship to total serum IgE levels and blood eosinophils during and out of the pollen season. Clin Exp Allergy 1997;27:1052-9.

14. Heinzerling L, Frew AJ, Bindslev-Jensen C, Bonini S, Bousquet J, Bresciani M, et al. Standard skin prick testing and sensitization to inhalant allergens across Europe-a survey from the GALEN network. Allergy 2005;60:1287-300.

15. Kerkhof M, Dubois AE, Postma DS, Schouten JP, de Monchy JG. Role and interpretation of total serum IgE measurements in the diagnosis of allergic airway disease in adults. Allergy 2003;58:905-11.

16. Bousquet J, Anto JM, Bachert C, Bousquet PJ, Colombo P, Crameri R, et al. Factors responsible for differences between asymptomatic subjects and patients presenting an IgE sensitization to allergens. A GA2LEN project. Allergy 2006;61:671-80.

17. Di Lorenzo G, Mansueto P, Melluso M, Candore G, Colombo A, Pellitteri ME, et al. Allergic rhinitis to grass pollen: measurement of inflammatory mediators of mast cell and eosinophils in native nasal fluid lavage and in serum out of and during pollen season. J Allergy Clin Immunol 1997;100:832-7.

18. Pastorello EA, Incorvaia C, Ortolani C, Bonini S, Canonica GW, Romagnani S, et al. Studies on the relationship between the level of specific IgE antibodies and the clinical expression of allergy: I. Definition of levels distinguishing patients with symptomatic from patients with asymptomatic allergy to common aeroallergens. J Allergy Clin Immunol 1995;96:580-7.

19. Jackola DR, Blumenthal MN, Rosenberg A. Evidence for two independent distributions of serum immunoglobulin $\mathrm{E}$ in atopic families: cognate and non-cognate IgE. Hum Immunol 2004;65:20-30.

20. Xystrakis E, Urry Z, Hawrylowicz CM. Regulatory T cell therapy as individualized medicine for asthma and allergy. Curr Opin Allergy Clin Immunol 2007;7: 535-41.

21. MacGlashan DW Jr, Bochner BS, Adelman DC, Jardieu PM, Togias A, McKenzieWhite J, et al. Down-regulation of Fc(epsilon)RI expression on human basophils during in vivo treatment of atopic patients with anti-IgE antibody. J Immunol 1997;158:1438-45. 


\section{METHODS}

\section{Immunotherapy intervention}

Dose administered of SCIT and SLIT. All the patients in this study tolerated the maximum dose indicated by the manufacturers' recommendations as follows. For SCIT, the maintenance dose was $0.8 \mathrm{~mL}$, which corresponds to $1.6 \mu \mathrm{g}$ of the major allergen of grass, $0.4 \mu \mathrm{g}$ of the major allergen of $P$ judaica, $9.6 \mu \mathrm{g}$ of the major allergen of $O$ europea, and $3.84 \mu \mathrm{g}$ of the major allergens of $D$ pteronyssinus and $D$ farinae. For SLIT, the maintenance dose was 60 drops $(1$ drop $=25 \mu \mathrm{L})$ administered 3 times a week on alternate days, which corresponds to $28.2 \mu \mathrm{g}$ of the major allergen of grass, $6.6 \mu \mathrm{g}$ of the major allergen of $P$ judaica, $169.2 \mu \mathrm{g}$ of the major allergen of $O$ europea, and $67.7 \mu \mathrm{g}$ of the major allergens of $D$ pteronyssinus and $D$ farinae. These doses were given monthly. Immunotherapy with grass, $P$ judaica, and $O$ europea pollen was not interrupted during the spring, but the dose was halved.

The use of a perennial schedule with grass, $P$ judaica, and $O$ europea pollen was well tolerated in our patients, as shown by the absence of side effects associated with administration during the spring, the peak period of these pollens, both in Sicily and in Veneto.

We did not find a relationship between allergen dosing, total dose received, and clinical response because all the patients received an equal dose.

Assessment of symptoms and medication use. For clinical control, with regard to the 3 outdoor allergens, we performed clinical control and evaluation of the VAS for symptoms at the end of the season, specifically in June for patients allergic to grass and $O$ europea and in September for patients allergic to $P$ judaica. For patients allergic to $D$ pteronyssinus and $D$ farinae, we performed clinical control and evaluation of the VAS for symptoms at the end of February because they are typically increased in autumn, after vacations, and during winter.

In Sicily, however, there is not a well-defined season for grass and $P$ judaica. Thus the use of medication and, perhaps, symptom scoring might be outside the pollen season and therefore might be relevant to the assessment of the efficacy of immunotherapy.
Evaluation of clinical response. The clinical response to ASI was evaluated with separate VASs for rhinitis and asthma. For rhinitis, the patients assessed their total nasal symptom scores (sneezing, rhinorrhea, nasal congestion, and nasal pruritis) yearly for 4 years with the VAS. Subjects were instructed that 0 indicated "nasal symptoms not at all bothersome" and that 100 indicated "nasal symptoms extremely bothersome." For asthma, the patients did the same, marking the VAS for all asthma symptoms yearly for 4 years (shortness of breath, chest tightness, cough, and wheezing). Each VAS was $100 \mathrm{~mm}$ horizontal with 0 and 100 on the left and right ends, respectively. Subjects were instructed that 0 indicated "no complaints of respiratory sensation, such as shortness of breath, chest tightness, and breathlessness" and 100 indicated "the worst complaints of respiratory symptoms imaginable." The distance between 0 and the marks the subjects made on the scale was measured with a Digimatic caliper (Mitutoyo, Kawasaki, Japan).

Rescue medication included an $\mathrm{H}_{1}$-antagonist for relief of rhinitis symptoms (loratadine [Claritin], $10 \mathrm{mg}$; Schering-Plough, Segrate, Italy) and a short-acting $\beta_{2}$-agonist for relief of asthma symptoms (salbutamol [Ventolin], $100 \mu \mathrm{g}$ per actuation of a metered-dose inhaler; GlaxoSmithKline, Naples, Italy). We asked patients to indicate the number of packages of loratadine tablets (20 tablets per package) taken in each year and the number of salbutamol inhalers used in each year. The scale of rescue medication was between 0 and 3 . The subjects were instructed that 0 indicated no rescue medication; 1 indicated a maximum of 1 package of loratadine $(\leq 20$ tablets per year; see Table E1) and 1 salbutamol inhaler $(\leq 20 \mathrm{mg} / \mathrm{y}) ; 2$ indicated a maximum of 3 packages of loratadine ( $\leq 60$ tablets per year) and a maximum of 4 salbutamol inhalers $(\leq 80 \mathrm{mg} / \mathrm{y})$; and 3 indicated 4 or more packages of loratadine and 5 or more salbutamol inhalers (Table E2).

Comparison between SCIT and SLIT in patients with effective clinical responses to ASI. The comparison between SCIT and SLIT in patients with an effective clinical response to ASI is shown in Table E3. Significant differences were noted only for atopic family history $(P<.0001)$ and serum s-IgE levels $(P=.02)$. 
TABLE E1. Short-acting $\boldsymbol{\beta}_{2}$-agonist used as rescue medication for asthmatic symptoms

\begin{tabular}{|c|c|c|c|c|}
\hline \multirow[b]{3}{*}{ Rescue medication } & \multicolumn{4}{|c|}{ No. of patients } \\
\hline & \multicolumn{2}{|c|}{$\begin{array}{l}\text { Ineffective clinical } \\
\text { response }(n=54)\end{array}$} & \multicolumn{2}{|c|}{$\begin{array}{l}\text { Effective clinical } \\
\text { response }(n=73)\end{array}$} \\
\hline & Before ASI & After ASI & Before ASI & After ASI \\
\hline 0 (no rescue medication) & 0 & 0 & 0 & 68 \\
\hline 1 (mild use) & 10 & 13 & 9 & 5 \\
\hline 2 (moderate use) & 22 & 30 & 35 & 0 \\
\hline
\end{tabular}


TABLE E2. $\mathrm{H}_{1}$-antagonist used as rescue medication for symptoms of rhinitis

\begin{tabular}{|c|c|c|c|c|}
\hline \multirow[b]{3}{*}{ Rescue medication } & \multicolumn{4}{|c|}{ No. of patients } \\
\hline & \multicolumn{2}{|c|}{$\begin{array}{l}\text { Ineffective clinical } \\
\text { response }(n=80)\end{array}$} & \multicolumn{2}{|c|}{$\begin{array}{l}\text { Effective clinical } \\
\text { response }(n=72)\end{array}$} \\
\hline & Before ASI & After ASI & Before ASI & After ASI \\
\hline 0 (no rescue medication) & 0 & 0 & 0 & 0 \\
\hline 1 (mild use) & 0 & 0 & 0 & 70 \\
\hline 2 (moderate use) & 22 & 30 & 21 & 2 \\
\hline
\end{tabular}


TABLE E3. Patients with effective clinical responses to ASI: Comparison between SCIT and SLIT

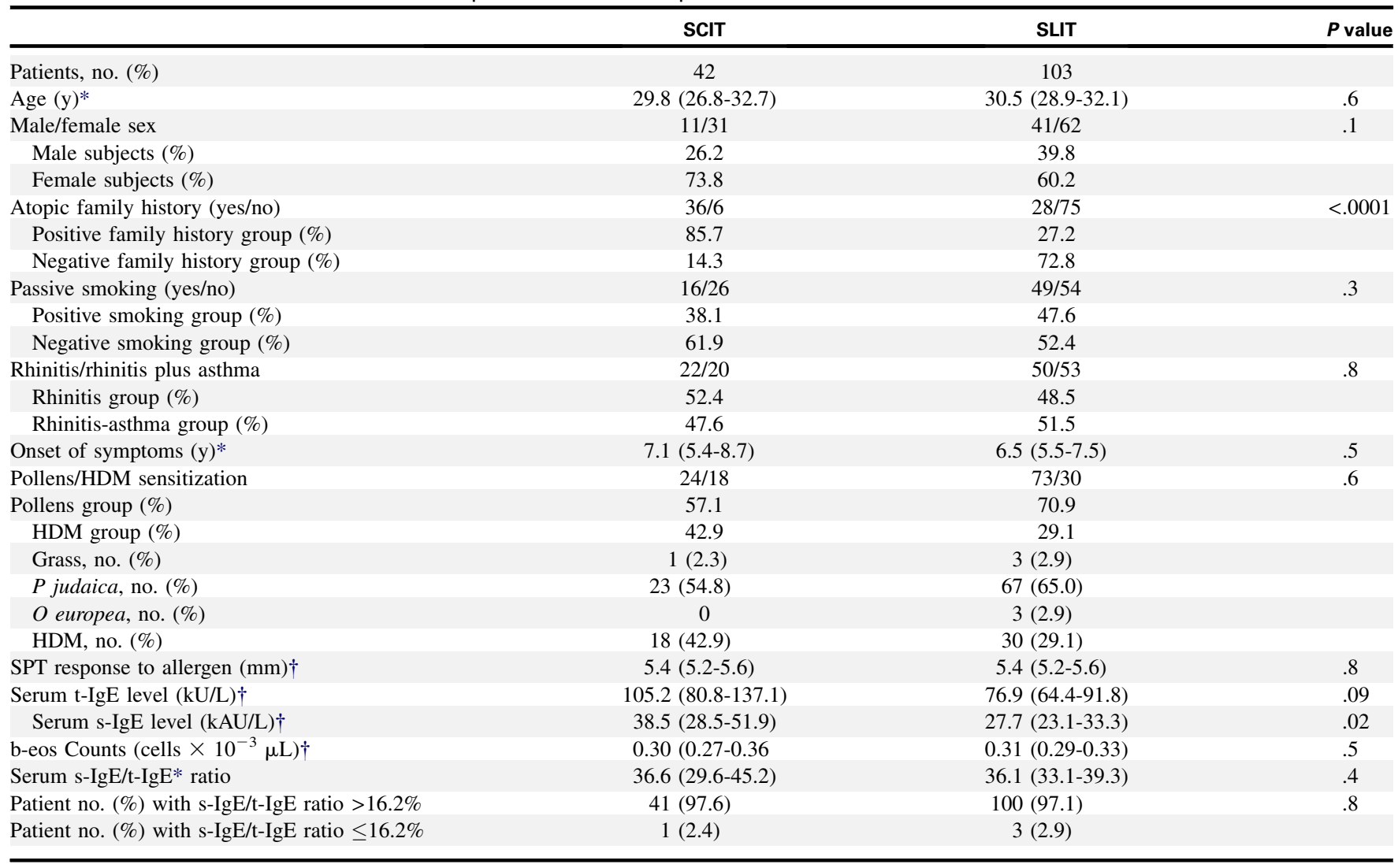

SPT, Skin prick test.

*Mean $(95 \% \mathrm{CI})$

†Geometric mean after logarithmic transformation $(95 \% \mathrm{CI})$. 